ARTICLE

\title{
Monte Carlo Code for the Damage of Bio-Molecules Irradiated by X-Ray Free Electron Lasers: Incorporation of Election Impact Ionization Processes
}

\author{
Kengo MORIBAYASHI * \\ Japan Atomic Energy Agency, 8-1-7, Umemidai, Kizugawa-city, 619-0215, Japan
}

\begin{abstract}
In this paper, electron impact ionization processes are incorporated in our Monte Carlo (MC) code for the calculation of the damage of the Bio-Molecules by the irradiation of X-Ray free electron lasers (XFELs). The study of this damage is useful for the analysis of three-dimensional structure of the Bio-Molecules using X-Ray free electron lasers because the damage appears as a noise for this analysis. The X-Ray absorption and Compton scattering processes take place after the X-Rays irradiate the target. Then, an electron is produced from atoms and moves in the target. This electron also gives rise to an electron impact ionization process for the other atoms or ions. It is assumed that electron impact ionization processes occur only when the electrons cross a cross section, which is located at the place of the atomic nucleus and is perpendicular to the direction of the electron velocity. The X-Ray flux, wavelength, and pulses of XFEL light pulses treated here are $10^{20-21} / \mathrm{pulse} / \mathrm{mm}^{2}, 0.1 \mathrm{~nm}$, and $10 \mathrm{fs}$, respectively. We compare the frequencies of photo-electron impact ionization processes calculated by our MC code with those by rate equations. The relationship of these frequencies with shapes of targets using various ellipsoids as a target is discussed.
\end{abstract}

KEYWORDS: X-Ray damage of Bio-Molecules, X-Ray free electron lasers, Monte Carlo, photo-electron impact ionization, shape dependence

\section{Introduction}

The study of the damage and the destruction of Bio-Molecules due to the irradiation of X-Ray free electron laser (XFEL) light pulses is indispensable for the analysis of three-dimensional (3D) structures using non-crystallized single Bio-Molecules, which is one of the most noteworthy application of XFEL light pulses. ${ }^{1-13)}$ We define the damage and the destruction as the ionization and the movement of atoms in a target, respectively. This comes from the fact that places of the atoms are and are not changed due to the movement and the ionization, respectively. The change of the places means that the reconstruction of the $3 \mathrm{D}$ structure cannot be executed. The damage and the destruction mainly occur through the following occurrences: (i) the atoms in the target are ionized through the X-Ray absorption or Compton scattering. (ii) From these ionization processes, free electrons, quasi-free electrons and ions are produced and move, where we define 'a free electron' and 'a quasi-free electron' as an electron, which leaves from an atom, outside and inside the target, respectively. (iii) Quasi-free electrons promote the ionization of the other atoms through electron impact ionization processes. (iv) Other ionization processes, such as Auger, also occur.

Before experiments of 3D structure start, simulations for the damage and the destruction play an important role. We have been developing the Monte Carlo (MC) code for the calculation of the damage of Bio-Molecules or clusters due

*Corresponding author, E-mail:moribayashi.kengo@jaea.go.jp to the irradiation of XFEL light pulses. ${ }^{9,11)}$ In this paper, we incorporate electron impact ionization processes in our MC code and discuss the relationship of shapes of targets with electron impact ionization processes by treating various ellipsoids as the target.

\section{Method of Calculations}

We have developed MC code, which is different from ordinary particle simulation codes. In our MC code, we can calculate the change of the electronic states of atoms in clusters or Bio-Molecules due to some atomic processes such as $\mathrm{X}$-Ray absorption ionization processes and the movement of free and quasi-free electrons, which are produced from some ionization processes.

Here, we treat model carbon clusters with various shapes such as spheres and ellipsoids at a solid density $(3 \times$ $\left.10^{22} / \mathrm{cm}^{3}\right)$. We decide places inside and outside the target from the number and the density of atoms. Then, the places of the atoms are assigned randomly on the condition that they are located inside the target and that lengths among the atoms are larger than $0.3 \mathrm{~nm}$.

For the parameters of XFEL light pulses, it is estimated that X-Ray fluxes around $10^{20}$ photons $/ \mathrm{pulse} / \mathrm{mm}^{2}$ and wavelength around $0.1 \mathrm{~nm}$ are required. ${ }^{1,4,6)}$ In this paper, we treat X-Ray fluxes of $10^{20}$ to $10^{21}$ photons $/ \mathrm{pulse} / \mathrm{mm}^{2}$, a wavelength of $0.1 \mathrm{~nm}$, a pulse of $10 \mathrm{fs}$, and the number of atoms of $100-8,000$.

Ionization processes treated here are the X-Ray absorption (e.g., $\mathrm{C}+h v \rightarrow \mathrm{C}^{+}+\mathrm{e}^{-}$), Compton scattering (e.g., $\mathrm{C}+$ 
$\left.h v \rightarrow \mathrm{C}^{+}+\mathrm{e}^{-}+h v^{\prime}\right)$, the electron impact ionization (e.g., $\mathrm{C}$ $+\mathrm{e}^{-} \rightarrow \mathrm{C}^{+}+2 \mathrm{e}^{-}$), and Auger (e.g., $\mathrm{C}^{+^{*}} \rightarrow \mathrm{C}^{2+}+\mathrm{e}^{-}$), where $h v$ and $h v^{\prime}$ are the X-Ray energies before and after the process occurs, respectively. We calculate the change of both of ionized and excited states of the atoms and the production of free and quasi-free electrons using rates or cross sections of these ionization processes as a function of times. We use the same rates or cross sections as those given by ourselves, ${ }^{7,10)}$ Bell el al., ${ }^{14)}$ and Henke et al.: ${ }^{15)}$ The X-Ray absorption cross sections $\left(\sigma_{x a}\right)$ are calculated by Cowan code $^{16)}$ where Hartree-Fock and Configuration Interaction methods are employed. On the other hand, the cross sections of Compton scattering $\left(\sigma_{C S}\right)$ are determined by the Klein-Nishina formula. ${ }^{17,18)}$ Then, the rates of the X-Ray absorption $\left(R_{x a}\right)$ and Compton scattering $\left(R_{C S}\right)$ are given by $R_{x a}=I \sigma_{x a} / h \mathrm{v}$ and $R_{C S}=I \sigma_{C s} / h \mathrm{v}$, respectively, where $I$ is the intensity of the X-Rays. ${ }^{19)}$ Auger rates are calculated using Cowan code. ${ }^{16)}$ The cross sections of the electron impact ionization processes $\left(\sigma_{e}\right)$ are given in the data given by Bell et al.. ${ }^{14)}$ For the rate equations, we use the rates for the electron impact ionization processes, that is, $R_{e}=N_{e} \sigma_{e} v_{e}$, where $N_{e}$ and $v_{e}$ are the electron density and the electron velocity, respectively. ${ }^{19)}$ In our MC code, we employ $\sigma_{e}$ as mentioned later.

The initial energies and velocities of electrons produced from these ionization processes should be mentioned because they contribute significantly not only to the movement of free and quasi-free electrons but also to the treatment of electron impact ionization processes. (i) The X-Ray absorption processes: The initial electron energy corresponds to the value that subtracted a bound energy $\left(E_{B}\right)$ of atoms or ions from the X-Ray energy. Since the X-Ray energy treated here is much larger than $E_{B}$ of $\mathrm{H}, \mathrm{C}, \mathrm{N}$, and $\mathrm{O}$, which are main elements of Bio-Molecules, the initial electron energy is almost the same as the X-Ray energy. (ii) The Compton scattering: The values of the scattering angles, which determine $h v$, are solved randomly by treating the Klein-Nishina formula ${ }^{17,18)}$ as a weighting factor and the initial electron energy is $h v-h v^{\prime}$. (iii) Auger: We employed the initial electron energy calculated by Cowan code. ${ }^{16)}$ (iv) Electron impact ionization processes: We calculate the initial electron energy from the binary encounter dipole (BED) theory ${ }^{10,20)}$ or use of the data given by Nakazaki et al.. ${ }^{21)}$ After the initial electron energy is determined, the initial direction of the electron velocity is given randomly except for that due to Compton scattering. In Compton scattering, the initial direction is determined from the electron energy, the scattering angles, and the momentum conservation law.

We mainly employ our MC code. However, in order to confirm the accuracy of the calculation by our MC code, we also show the results calculated by the rate equations ${ }^{7,19,22)}$ aimed at the comparison.

The X-Ray absorption, Auger, and Compton scattering processes are treated using our $\mathrm{MC}$ as follows: ${ }^{23)}$ (i) just when an XFEL begins to irradiate a target, we start the calculation and set the time of $t=0$. We also set the neutral and the ground states for ionized and excited states of all atoms in the target, respectively. (ii) We prepare the transition rates

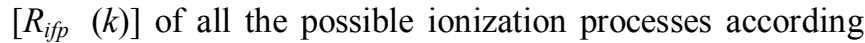
to the ionized and excited states of all the atoms and random numbers $\left[N_{R}(k)\right]$. One random number is given to each atom at the time interval between $t$ and $t+\Delta t$, where $R_{i f p} \quad(k)$ and $N_{R}(k)$ are the transition rate from the $i$ 'th state to the $f$ 'th one due to the $p$ 'th ionization process and the random number given to the $k^{\prime}$ th atom, respectively. (iii) Only when

$$
\sum_{p} \sum_{f} R_{i f p}(k) \Delta t<N_{R}(k)
$$

one process for the $k^{\prime}$ th atom occurs. When Eq. (1) is satisfied, the state where the ionization occurs is chosen randomly among all the possible transitions using the respective $R_{i f p}(k)$ as weighting factors. (iv) The value of $t$ increases by $\Delta t$ and procedures (ii) and (iii) are executed. (v) We reiterate procedures (ii) - (iv) until the XFEL light pulse passes through the target.

As for the electron impact ionization process, the similar method as that treated by Jurke et $a l^{2,3)}$ is employed. It is judged that the process occurs only when a quasi-free electron crosses the area of a cross section according to an ionized state of an atom or an ion. The center of the cross section is located at the place of the atomic nucleus and the cross section is perpendicular to the direction of the electron velocity.

It should be noted that the production and the movement of electrons depend on the initial values of the random numbers (seeds) and that we can demonstrate the calculations of the damage and the electron distributions for different pulses using different initial seeds for the random number generated. We will show the results averaged by a few hundred pulses in Section III.

\section{Results and Discussions}

Figure 1 shows the distribution of $v_{z}$ with respect to $\left(v_{x}^{2}+\right.$ $\left.v_{y}{ }^{2}\right)^{1 / 2}$ where $v_{x}, v_{y}$, and $v_{z}$ are $x, y$, and $z$-components of the initial electron velocities of electrons produced from (a) X-Ray absorption, (b) Compton scattering, and (c) Auger processes, respectively and we adopt the $z$-axis as the incident direction of XFEL light pulses. We treat a few hundreds pulses as mentioned in Section II and when one process occurs, one point in Fig. 1 is added. For X-Ray absorption and Auger processes, electrons are emitted in the isotropic directions. On the other hand, Compton scattering produces an electron in front. The energies of electrons $\left[\propto\left(v_{\mathrm{x}}{ }^{2}+v_{\mathrm{y}}{ }^{2}+\right.\right.$ $\left.v_{\mathrm{z}}^{2}\right)$ ] due to $\mathrm{X}$-Ray absorption remain almost constant values and are larger than the energies of electrons produced from Compton and Auger processes, while various energies exist in the electrons produced from Auger and Compton scattering. In the distribution produced from Compton scattering [see Fig. 1(b)], there are two lines, which are produced from inner-shell (red points) ionization and outer-shell ionization (blue ones), respectively.

A photo-electron is defined as an electron produced from the X-Ray absorption. Photo-electrons often prompt the ionization of the other atoms in the target through the electron impact ionization processes before they escape from the target. Here, we compare the frequency $\left(F_{p e i}\right)$ of photo-electron 
impact ionization processes calculated by our MC code with that by the rate equations in order to confirm the accuracy of the electron impact ionization processes calculated by our $\mathrm{MC}$ code. When we treat the photo-electron impact ionization in the rate equation calculations, we need to calculate the averaged track length $\left(r_{t a}\right)$. Here, a track length $\left(r_{t}\right)$ is defined as a distance over which a photo-electron moves from the production of it to the point where it escapes from the target. The calculation of $r_{t a}$ is executed as follows. (i) We set the place of the production and the directions of the velocity for a photo-electron randomly. (ii) At each site where the photo-ionization is produced, $r_{t}$ is calculated. We reiterate procedures (i) and (ii). (iii) We estimate $r_{t a}$ by averaging over many values of $r_{t}$. As a result, we have found $r_{t a}$ $\sim 0.75 \times r_{0}$ for spherical targets, where $r_{0}$ is the radius of the target.

Figure 2 shows the comparison of the values of $F_{p e i}$ of photo-electron impact ionization processes calculated by our $\mathrm{MC}$ code with that by the rate equations for spherical targets with the radius $\left(r_{0}\right)$ of $1-4 \mathrm{~nm}(100-8,000$ atoms $)$ and $\mathrm{X}$-Ray fluxes of $10^{20}-10^{21}$ photons/pulse $/ \mathrm{mm}^{2}$. From Fig. 2, we have found agreement of $80 \%$ to $90 \%$ for both of the values calculated by the two methods.

In order to study the shape dependence on $F_{p e i}$, we employ not only spherical targets but also various ellipsoids. Figure 3 shows $F_{p e i}$ calculated by our MC code and $r_{t a}$ as a function of $l_{r}$, where $l_{r}$ is the ratio of the length of the major axis with that of the minor one of a ellipsoid. We treat the number of atoms of 2,000 ( $r_{0}$ is $\left.2 \mathrm{~nm}\right)$ in the target and $\mathrm{X}$-Ray flux of $10^{20}$ photons/pulse/ $\mathrm{mm}^{2}$ in Fig. 3. Both values of $F_{p e i}$ and $r_{t a}$ are normalized at $l_{r}=1$. We have found that $r_{t a}$ agrees well with $F_{p e i}$ as a function of $l_{r}$. We have found from Fig. 3 that $F_{p e i}$ decreases as $l_{r}$ becomes smaller. Namely, the damage due to photo-electron impact ionization processes becomes smaller according to the increase of $l_{r}$, that is, we may be able to reduce the damage, which appears as a noise in the analysis of 3D structure, by choosing a target with larger value of $l_{r}$. When we employ rate equations, we need to calculate $r_{t a}$ for the target treated there and the place of the production of photo-electrons should be located at $r_{s}=r_{0}$.

\section{Summary}

We have corporate electron impact ionization processes in our Monte Carlo (MC) code for the calculation of the damage of Bio-Molecules or clusters. We compare the frequency of photo-electron impact ionization calculated by our MC code with those by the rate equations and we have found the agreement of more than $80 \%$ between them. The frequency of photo-electron impact ionization processes decreases as $l_{r}$ becomes larger, where $l_{r}$ is the ratio of the lengths of major axis with that of a minor one in the ellipsoid. Namely, the damage due to the photo-electron impact ionization processes becomes smaller according to the increase of $l_{r}$, that is, we may reduce the damage, which appears as a noise in the analysis of the 3D structure, by choosing a target with larger values of $l_{r}$.

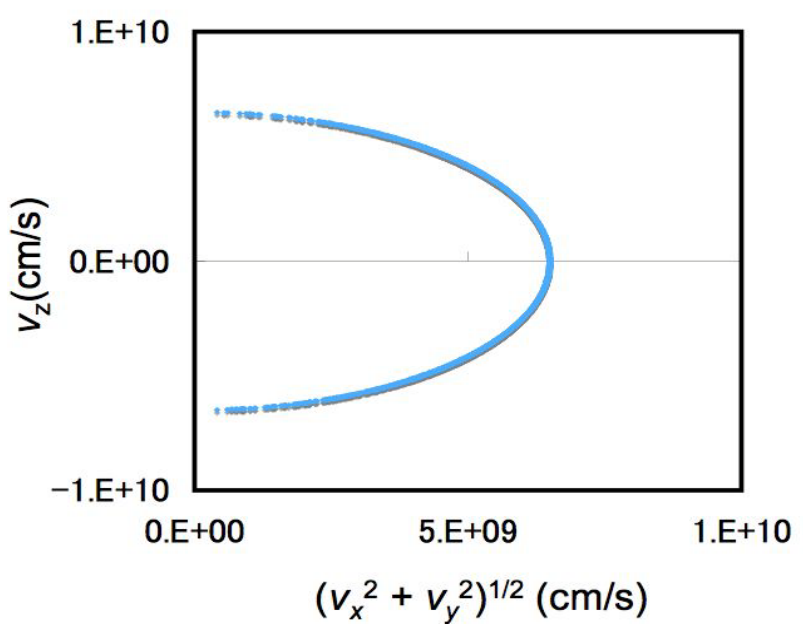

(a)

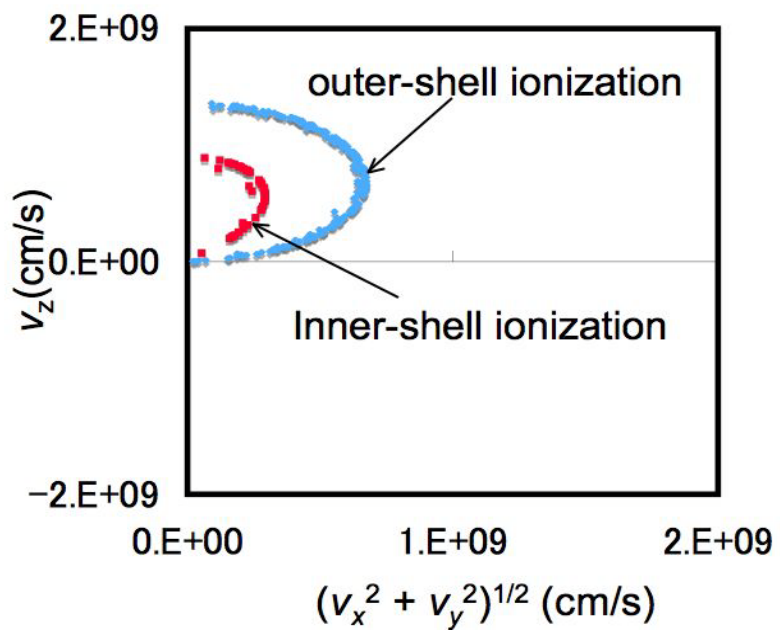

(b)

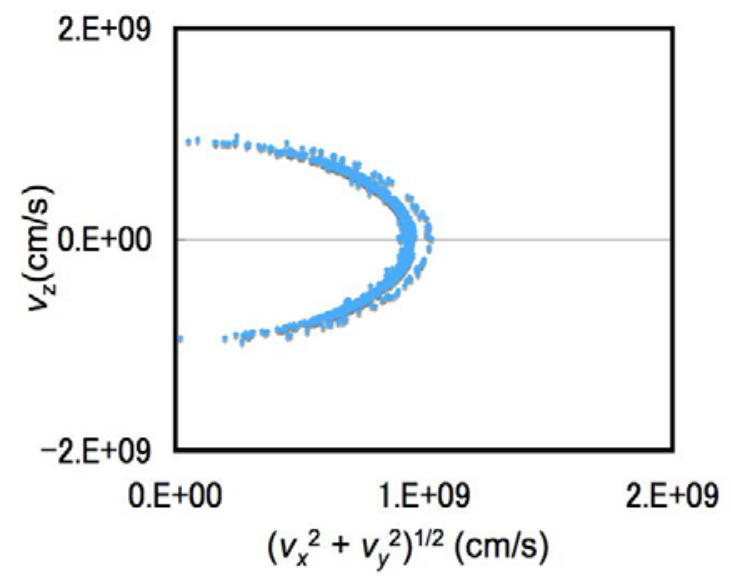

(c)

Fig. 1 The distribution of $v_{z}$ with $\left(v_{x}^{2}+v_{y}^{2}\right)^{1 / 2}$ of the initial velocities of electrons produced from (a) X-Ray absorption, (b) Compton scattering, and (c) Auger processes

\section{Acknowledgements}

We wish to thank Prof. N. Go, Drs. H. Kono, T. Kai, A. Tokushisa, J. Koga, and M. Yamagiwa for their useful discussions. For the calculation of atomic data, we employ 


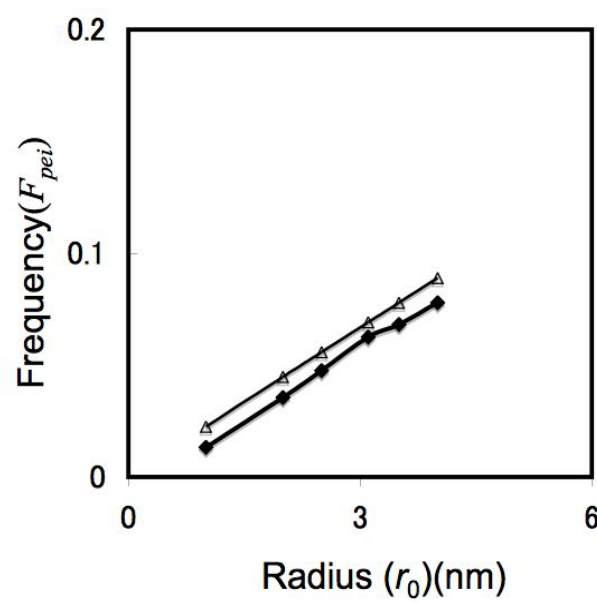

(a)

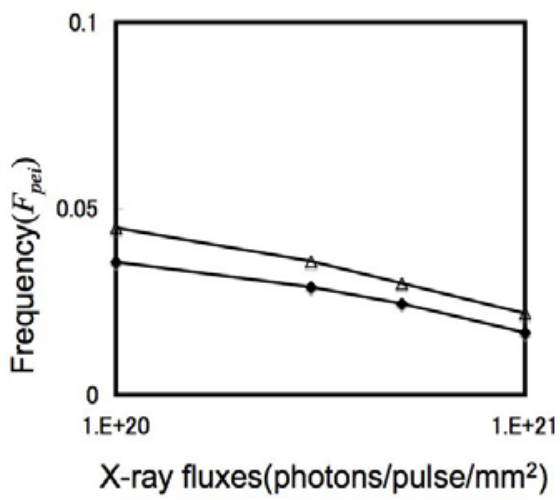

(b)

Fig. 2 Frequencies of photo-electron impact ionization processes as functions of (a) radius of the target and (b) $\mathrm{X}$-Ray fluxes. The methods of calculations are rate equations $(\triangle)$ and Monte Carlo $(\bullet)$.

Cowan's code. This study has been supported by the 'X-Ray Free Electron Laser Utilization Research Project' of the Ministry of Education, Culture, Sports, Science and Technology of Japan (MEXT).

\section{References}

1) R. Neutze, R. Wouts, D. Spoel, E. Weckert, J. Hajdu, "Potential for biomolecular imaging with femtosecond X-Ray pulses," Nature, 406, 752-757 (2000).

2) Z. Jurek, G. Faigel, M. Tegze, "Dynamics in a cluster under the influence of intense femtosecond hard X-Ray pulses, " Eur. Phys. J., D 29, 217-229 (2004).

3) Z. Jurek, G. Faigel, "The effect of tamper layer on the explosion dynamics of atom clusters," Eur. Phys. J., D50, 35-43 (2008).

4) S. P. Hau-Riege, R. London, A. Szoke, "Dynamics of biological molecules irradiated by short X-Ray pulses," Phys. Rev., E69, 051906 (2004).

5) S. P. Hau-Riege, R. A. London, H. N. Chapman, A. Szoke, N. Timneanu, "Encapsulation and diffraction-pattern- correction methods to reduce the effect of damage in X-Ray diffraction imaging of single biological molecules," Phys. Rev. Lett., 98, 198302 (2007).

6) K. Moribayashi, T. Kai, "Atomic processes for the damage on Bio-Molecules irradiated by XFEL,” J. Phys. Conf. Ser., 163,

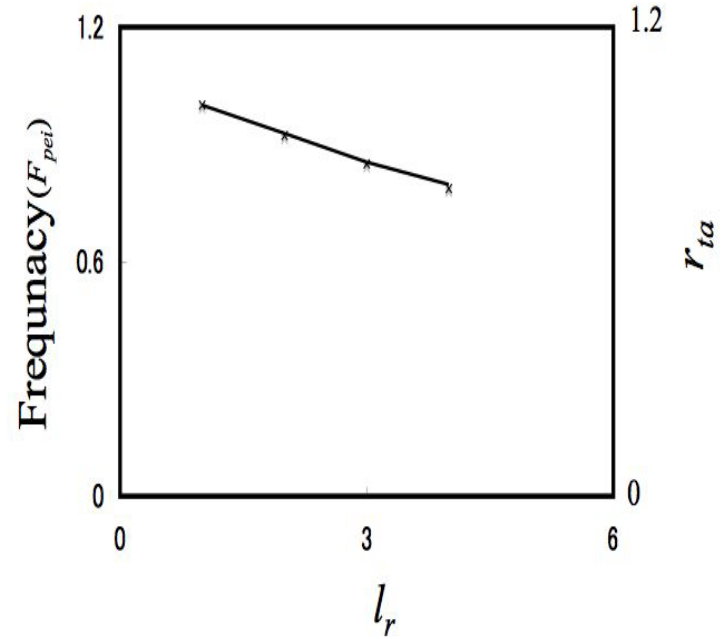

Fig. 3 Frequency $\left(F_{p e i}\right)(-)$ and $r_{t a}(\times)$ as a function of $l_{r}$

012097 (2009).

7) K. Moribayashi, "Application of XFEL to the measurement of $\mathrm{X}$-Ray flux irradiating Bio-Molecules by using X-Ray emission from hollow atoms produced from multiple X-Ray absorptions," J. Phys., B41, 085602 (2008).

8) B. Ziaja, A. R B. de Castro, E. Weckert, T. Möller, "Modelling dynamics of samples exposed to free-electron-laser radiation with Boltzmann equations," Eur. Phys. J., D40, 465480 (2006).

9) K. Moribayashi, “Application of photoelectron spectroscopy to the measurement of the flux of X-Ray free-electron lasers irradiating clusters or Bio-Molecules," Phys. Rev., A80, 025403 (2009).

10) T. Kai, K. Moribayashi, "Effect of electron-impact ionization in damage of Bio-Molecules irradiated by XFEL," J. Phys. Conf. Ser., 163, 012035 (2009).

11) K. Moribayashi, "Spherically symmetric models on the X-Ray damage and the movement of electrons produced in non-spherically symmetric targets such as Bio-Molecules," $J$. Phys., B43, 165602 (2010).

12) T. Kai, "Single-differential and integral cross sections for electron-impact ionization for the damage of carbon clusters irradiated with X-Ray free-electron lasers," Phys. Rev., A81, 023201 (2010).

13) T. Nakamura, Y. Fukuda, Y. Kishimoto, "Ionization dynamics of cluster targets irradiated by X-Ray free-electron-laser light," Phys. Rev., A80, 053202 (2009).

14) K. L. Bell, H. B. Gilbody, J. G. Hughes, A. E. Kingston, F. J. Smith, "Recommended data on the electron impact ionization of light atoms and ions," J. Phys. Chem. Ref. Data, 12, 891-916 (1983).

15) B. L. Henke, E. M., Gullikson, J. C. Davis, "X-Ray interactions: photoabsorption, scattering, transmission, and reflection at $E=50-30,000 \mathrm{eV}, Z=1-92, "$ Atomic data \& Nuc. Data Tables, 54, 181-342 (1993).

16) R. D. Cowan, "Theoretical calculation of atomic spectra using digital computers," J. Opt. Soc. Am., 58, 808-818 (1968).

17) V. O. Klein, Y. Nishina, "Über die Streuung von Strahlung durch freie elektronen nach der neuen relativistischen quntendynamik von Dirac," Z. Phys., 52, 853-868 (1929). 
18) G. R. Blumenthal, R. J. Gould, "Bremsstrahlung, synchrotron radiation, and Compton scattering of high- energy electrons traversing dilute gases," Rev. Mod. Phys., 42, 237-270 (1970).

19) K. Moribayashi, A. Sasaki, T. Tajima, "Ultrafast X-Ray processes wit hollow atoms," Phys. Rev., A58, 2007-2015 (1998).

20) Y. K. Kim, J. P. Santos, F. Parente, "Cross sections for singly differential and total ionization of helium by electron impact," Phys. Rev., A62, 052710 (2000).

21) S. Nakazaki, M. Nakashima, H. Takebe, K. Takayanagi
"Energy distribution of secondary electrons in electron- impact ionization of hydrogen-like ions," J. Phys. Soc. Japan, 60, 1565-1571 (1991).

22) K. Moribayashi, "Multiply inner-shell excited states produced through multiple X-Ray absorption relevant to X-Ray pulses," Phys. Rev., A76, 042705 (2007).

23) K. Moribayashi, "Comparison of the stopping powers calculated by using rate equation with those by Monte Carlo method," J. Phys. Conf. Ser., 58, 192-194 (2007). 